\title{
Diacronie
}

Studi di Storia Contemporanea

$N^{\circ} 17,1 \mid 2014$

Periferie. Cultura, economia, politica

\section{Martha C. Nussbaum, La nuova intolleranza. Superare la paura dell'Islam e vivere in una società più libera}

\section{Luca Zuccolo}

\section{Q OpenEdition}

Edizione digitale

URL: http://journals.openedition.org/diacronie/1144

DOI: 10.4000/diacronie. 1144

ISSN: 2038-0925

Editore

Association culturelle Diacronie

Notizia bibliografica digitale

Luca Zuccolo, « Martha C. Nussbaum, La nuova intolleranza. Superare la paura dell'Islam e vivere in una società più libera », Diacronie [Online], № 17, 1 | 2014, documento 14, Messo online il 01 mars 2014, consultato il 23 septembre 2020. URL : http://journals.openedition.org/diacronie/1144; DOI : https:// doi.org/10.4000/diacronie. 1144 


\section{Diacronie}

N. 17 | 1|2014 Periferie. Cultura, economia, politica

14/

\section{RECENSIONE:}

Martha C. NUSSBAUM, La nuova intolleranza. Superare la paura dell'Islam e vivere in una società più libera, Milano, Il Saggiatore, 259 pp.

a cura di Luca ZUCCOLO *

«L'ignoranza - vale a dire la paura dell'ignoto - è l'origine del pregiudizio più odioso» ${ }^{1}$. In questa affermazione si può riassumere il nuovo saggio di Martha Nussbaum, la quale intrecciando filosofia e storia ai più recenti fenomeni di intolleranza traccia una lucida analisi della paura religiosa e, in modo particolare, della paura dell'Islam.

Studiosa di filosofia e autrice del saggio Disgusto e Umanità (2011)², Nussbaum si cimenta in un'analisi della paura umana del diverso, incentrando la sua tesi sulla religione, sul rapporto Stato-religione e sull'approccio alla religione islamica e al mondo musulmano, come nuovo bersaglio delle paure occidentali. Sebbene riporti anche esempi sul rapporto con le religioni in India e sulla tolleranza in Europa, l'analisi dell'autrice si concentra essenzialmente sugli Stati Uniti. Tuttavia, come ricorda Stefano Rodotà nella sua prefazione:

il tema della paura va ben al di là degli schemi che hanno abitualmente accompagnato la fittissima bibliografia sulla paura americana dopo l'11 settembre, non a caso mai richiamata da Martha Nussbaum. Rivolta com'è alla paura dell'Islam, la sua analisi indaga nel profondo comportamenti e strutture sociali che mettono a rischio le condizioni stesse della libertà. Manifesta il bisogno di apprestare strumenti che possano portarci oltre gli scontri di civiltà, e le pessime

\footnotetext{
${ }^{1}$ NUSSBAUM, Martha C., La nuova intolleranza. Superare la paura dell'Islam e vivere in una società più libera, Milano, Il Saggiatore, p. 212.

${ }^{2}$ NUSSBAUM, Martha C., Disgusto e Umanità, Milano, Il Saggiatore, 2011.
} 
politiche che la perdurante scelta di questo punto di vista incessantemente produces.

Se il punto di partenza del nuovo studio di Nussbaum e di questo saggio è stata la proposta europea di bandire il burqa, lo scopo finale che l'autrice si propone, tuttavia, è molto più ampio e va al di là della tolleranza religiosa stricto sensu:

conosci te stesso in modo da poter uscire al di fuori della tua persona, servire la giustizia e promuovere la pace ${ }^{4}$.

Un messaggio sviscerato e ripetuto più volte nel testo, il quale con un'analisi chiara e accessibile e una struttura agile e di facile comprensione si presenta come un buon saggio per gli specialisti, ma anche per il pubblico non specialistico di lettori, a cui è specificamente indirizzato. Infatti, se la paura è il core topic del saggio altrettanto rilevante è l'intenzione dell'autrice di coinvolgere e ispirare nei suoi lettori un cambiamento di prospettiva, che si può ottenere innanzitutto con «un bel po' di storia e di informazioni contestuali corrette»5. Informazioni che l'autrice non solo invita a ricercare prima di dispensare giudizi e pregiudizi, ma che propone essa stessa all'interno dell'impianto testuale, alternandole a una teorizzazione filosofica ed etica molto stimolante, sebbene a volte un po' troppo autocelebrativa nei riguardi degli Stati Uniti.

Il campo d'analisi nordamericano, infatti, emerge fin dal primo capitolo dove, in chiave introduttiva, Nussbaum descrive i differenti atteggiamenti e le diverse risposte date da Stati Uniti ed Europa relativamente al tema religioso e all'Islam. Dopo aver enunciato i tre elementi su cui si concentrerà la sua analisi etico-filosofica - principi politici egalitari, coerenza analitica e immaginazione (sviluppo degli "occhi interiori”) l'autrice passa in rassegna alcuni dei principali nodi contemporanei legati alla tolleranza religiosa e al rapporto delle nostre società con l'Islam. Mette a confronto le polemiche e i divieti europei contro il burqa e i minareti con il diverso approccio Statunitense al foulard e alla Sharia, concludendo la sua analisi preliminare con il tema dell'identità nazionale e la sua diversa declinazione in Europa (omogeneità) e Stati Uniti (appartenenza).

Il secondo capitolo, invece, si concentra sulla paura descrivendola come un'emozione narcisistica. Attraverso lo studio dei filosofi greci e moderni e l'analisi di

3 RODOTÀ, Stefano, Prefazione, in NUSSBAUM, Martha C., La nuova intolleranza, cit., p. 11.

${ }_{4}^{4}$ NUSSBAUM, Martha C., La nuova intolleranza, cit., p. 17.

5 Ibidem, p. 217. 
esempi "classici" dell'antisemitismo come i Protocolli dei savi di Sion Nussbaum propone un'analisi dettagliata della paura a partire dai suoi aspetti biologici per poi passare a quelli culturali e retorici e, infine, ai suoi aspetti psicologici, in cui sottolinea i principali modi in cui essa si sviluppa: la disponibilità euristica ${ }^{6}$, la cascata reputazionale o informazionale ${ }^{7} \mathrm{e}$ il disgusto proiettivo $^{8}$. La paura, presentata come una tendenza biologica innata, tuttavia, non viene rigettata come eminentemente negativa. Per spiegare la duplicità di questo sentimento e il suo essere solo potenzialmente deleterio, l'autrice propone due serie di esempi. La paura razionale giustificata come forma di protezione viene rappresentata dalla tecnica del profiling nelle procedure di controllo aereoportuali e dalle misure prese per contrastare l'uragano Irene. La paura "irrazionale", invece, viene analizzata attraverso due esempi strettamente legati alla religione islamica: il divieto di costruire minareti in Svizzera e gli omicidi di Utøya in Norvegia.

Riprendendo le fila di quanto descritto nei primi due capitoli su religione e paura, nel terzo capitolo Nussbaum entra nel core topic del suo saggio proponendo un'analisi dettagliata del primo dei suoi tre principi fondamentali: la necessità di avere buoni principi di eguaglianza. Il capitolo si incentra sui diritti e sulla dignità e propone un'analisi di alcuni significativi casi giuridici americani relativi al tema della tolleranza religiosa. Casi analizzati confrontando la teoria del filosofo inglese John Locke e quella accomodazionista formulata dal filosofo anglo-americano Roger Williams nel XVII secolo. Il capitolo si conclude con alcune considerazioni sulle attuali relazioni tra Stato e religione e sull'importanza dell'eguaglianza per tutti i cittadini.

L'analisi dei tre principi di Nussbaum prosegue nel quarto capitolo, dove l'autrice esamina, partendo da alcuni dialoghi platonici per poi passare all'approccio cristianokantiano, il tema della coerenza e della vita esaminata. Fin dal titolo dato al capitolo La pagliuzza nell'occhio di mio fratello - l'autrice spiega qual è il suo obiettivo, ovvero criticare alcuni atteggiamenti distorti che le società occidentali odierne hanno nei

\footnotetext{
${ }^{6}$ «Se possiamo facilmente richiamare alla memoria un problema impresso in modo vivido nella nostra esperienza, saremo portati a sopravvalutarne l'importanza. [...] Se le persone sentono parlare molto di un determinato problema [...] tenderanno a pensare che quel pericolo sia più significativo di quanto non sia e a sottovalutare il pericolo di altri fenomeni che non appaiono altrettanto vividi e restano sullo sfondo». NUSSBAUM, Martha C., La nuova intolleranza, cit., p. 47.

7 «Gli individui rispondono al comportamento di altri individui uniformandosi a esso. Talvolta lo fanno in virtù della reputazione di quelle persone (la "cascata reputazionale"), e altre volte perché pensano che il comportamento degli altri fornisca loro nuove informazioni (la "cascata informazionale")». NUSSBAUM, Martha C., La nuova intolleranza, cit., p. 47.

8 «A queste possibili fonti di condizionamento possiamo aggiungere l'ansia che la maggior parte delle persone prova nei confronti della propria corporeità animale e delle vulnerabilità che questa comporta [...] il fenomeno che ho chiamato "disgusto proiettivo"». NUSSBAUM, Martha C., La nuova intolleranza, cit., p. 48.
} 
riguardi del diverso e della religione islamica. A sostegno della tesi teorica e degli esempi filosofici Nussbaum propone una dettagliata analisi dei motivi per cui il dibattito sul burqa è incoerente dimostrando con cinque motivazioni come in questi casi si parli spesso a sproposito e si dimentichi l’incongruenza, rispetto ai principi generali di tolleranza ed eguaglianza, di alcuni nostri atteggiamenti quotidiani. Tra queste motivazioni, di notevole rilievo - perché maggiormente notiamo la pagliuzza nell'occhio del nostro vicino senza accorgerci della trave nel nostro - quella relativa alla reificazione della donna. In questo paragrafo, infatti, Nussbaum sottolinea come i critici occidentali del burqa lo interpretino come sintomo e simbolo della reificazione della donna islamica, senza accorgersi o senza voler accorgersi che «le società moderne sono imbevute di simboli di supremazia maschile che trattano le donne come oggetti» 9 . Un tema oltremodo rilevante poiché mette in evidenza come i principi che Nussbaum propone per la tolleranza religiosa siano adattabili anche ad altri aspetti della vita quotidiana dei nostri tempi come, appunto, il rapporto con le donne o con gruppi sociali, come gli omosessuali, che sebbene siano sempre più accetti scontano ancora dei gravi problemi sociali dovuti all'ignoranza e a paure immotivate radicate nelle nostre società.

Il quinto capitolo conclude la descrizione dei tre principi analizzando quello decisivo e più difficile da ottenere: l'immaginazione empatica. L'analisi dell'importanza degli "occhi interni" si sviluppa attraverso lo studio di alcuni volumi, romanzi e libri per l'infanzia che, a partire dal XVII-XVIII secolo e dal saggio di Roger Williams sugli indiani e il modo "corretto" di interagire con loro, hanno proposto esempi significativi di ciò che significa tolleranza religiosa e sforzo di comprensione del diverso. Accanto al già ricordato saggio di Williams, quindi, troviamo l'opera teatrale Nathan de Weise di Lessing del 1770, il romanzo Daniel Deronda di George Eliot del 1876, entrambi incentrati sul tema degli ebrei e dell'antisemitismo - testimonianza di un problema critico per l'Europa e gli Stati Uniti non ancora del tutto superato e anticamera dell'attuale anti-islamismo - e, infine, i libri per l'infanzia della scrittrice americana Marguerite de Angeli (1889-1987) ${ }^{10}$.

Descritti analiticamente i tre principi attorno a cui ruota la sua tesi, Nussbaum passa alla pratica nel sesto capitolo descrivendo il caso Park51: il progetto di costruzione di un centro multiconfessionale gestito da un gruppo islamico nel Lower Manhattan a pochi isolati da Ground Zero. Esposti i fatti, descritto il dibattito acceso

\footnotetext{
9.Ibidem, p. 117.

1o DE ANGELI, Marguerite, Thee, Hannah!, Herald Press, Scottsdale, 2000; ID., Bright April, Doubleday, New York, 1947.
} 
che si è sviluppato attorno a questo progetto e presentati gli errori più evidenti dei costruttori e dei detrattori, l'autrice propone un esempio di come utilizzare i suoi convincimenti in un caso concreto, dimostrando come solo attraverso principi egualitari, coerenza e immaginazione empatica sia possibile superare le barriere pregiudiziali e raggiungere una vera tolleranza.

Nelle pagine conclusive del saggio Nussbaum riassume le sue analisi incentrando le sue conclusioni sulla necessità di combattere la politica della paura al fine di aprire il nostro sguardo all'altro e alla diversità senza ripiegarsi su noi stessi.

Il tema della intolleranza/tolleranza religiosa è senza dubbio contemporaneo e rilevante, ma allo stesso tempo ostico e spinoso; Nussbaum, tuttavia, riesce a proporlo in modo semplice e dettagliato, anche grazie ad un apparato di note completo e aggiornato ai più recenti avvenimenti, che le permette di rendere fruibile ad un ampio pubblico il tema e il suo saggio. Che il volume sia indirizzato anche ad un pubblico di non specialisti è senza dubbio meritorio, ma rende ancor più impegnativo l'obiettivo che si pone l'autrice. Mutare le prospettive sociali su argomenti così complessi, infatti, non è semplice, anche a causa della profondità con cui certi stereotipi sono radicati in noi e nelle nostre società. Nonostante ciò, il saggio di Nussbaum è un ottimo punto di partenza e conserva uno spirito analitico, in virtù del quale la sua lettura andrebbe caldamente consigliata. 


\section{* L'autore}

Luca Zuccolo è dottorando (PhD student) in Storia Contemporanea presso il SUM (Istituto Italiano di Scienze Umane) di Napoli dove sta sviluppando una ricerca sulla stampa francofona ottomana e la sua rappresentazione dell'Impero d'Oriente. Già dottore magistrale in Storia d'Europa (Bologna, 2008), il suo campo di ricerca si rivolge allo sviluppo della modernità durante l'ultimo secolo dell'Impero Ottomano, al confronto/scontro tra modernità e tradizione in un contesto cosmopolita e allo sviluppo dei movimenti sociali che hanno preparato l'avvento della società turca contemporanea.

URL: < http://www.studistorici.com/2010/12/o1/luca-zuccolo/>

\section{Per citare questo articolo:}

ZUCCOLO, Luca, «Recensione: Martha C. NUSSBAUM, La nuova intolleranza. Superare la paura dell'Islam e vivere in una società più libera, Milano, II Saggiatore, 259 pp.», Diacronie. Studi di Storia Contemporanea : Periferie. Cultura, economia, politica, 29/3/2014,

URL: < http://www.studistorici.com/2014/3/29/zuccolo_numero_17/ >

Diacronie Studi di Storia Contemporanea $\beta$ www.diacronie.it

Risorsa digitale indipendente a carattere storiografico. Uscita trimestrale.

redazione.diacronie@hotmail.it

Comitato di redazione: Jacopo Bassi - Luca Bufarale - Elisa Grandi - Deborah Paci - Fausto Pietrancosta - Matteo Tomasoni - Luca Zuccolo 